\title{
Stabilizing the electroweak vacuum by higher dimensional operators in a Higgs-Yukawa model
}

\section{Prasad Hegde}

National Taiwan University, Taipei, Taiwan

E-mail: phegde@physik.uni-bielefeld.de

Karl Jansen

NIC, Desy Zeuthen, Germany

E-mail: karl.jansen@desy.de

\section{C.-J. David Lin}

National Chiao Tung University, Hsinchu, Taiwan

E-mail: dlin@mail.nctu.edu.tw

\section{Attila Nagy* \\ Humboldt University Berlin; NIC, Desy Zeuthen, Germany \\ E-mail: nagy@physik.hu-berlin.de}

The Higgs boson discovery at the LHC with a mass of approximately $126 \mathrm{GeV}$ suggests, that the electroweak vacuum of the standard model may be metastable at very high energies. However, any new physics beyond the standard model can change this picture. We want to address this important question within a lattice Higgs-Yukawa model as the limit of the standard model (SM). In this framework we will probe the effect of a higher dimensional operator for which we take a $\left(\phi^{\dagger} \phi\right)^{3}$-term. Such a term could easily originate as a remnant of physics beyond the SM at very large scales.

As a first step we investigate the phase diagram of the model including such a $\left(\phi^{\dagger} \phi\right)^{3}$ operator. Exploratory results suggest the existence of regions in parameter space where first order transitions turn to second order ones, indicating the existence of a tri-critical line. We will explore the phase structure and the consequences for the stability of the SM, both analytically by investigating the constraint effective potential in lattice perturbation theory, and by studying the system non-perturbatively using lattice simulations.

31 st International Symposium on Lattice Field Theory LATTICE 2013

July 29 - August 3, 2013

Mainz, Germany

\footnotetext{
* Speaker.
} 
Stabilizing the electroweak vacuum by higher dimensional operators in a Higgs-Yukawa model Attila Nagy

\section{Introduction}

In 2012 a boson was discovered at the LHC with a mass around $126 \mathrm{GeV}$. Experimental results suggest, that this particle is compatible with the standard model Higgs boson [1,2]. Subsequent analyses following this discovery consolidated this interpretation, although a final proof is still missing. With the discovery of the Higgs boson and the measurement of its mass, the standard model (SM) is complete in the sense that in principle all its parameters are known. On the other hand, the SM cannot account for a number of phenomena observed in nature such as the quark mass hierarchy, the baryon asymmetry of the universe, the amount of CP violation and the existence of dark matter. Thus, at some yet unknown energy, the SM is expected to be replaced by some more comprehensive theory which can, hopefully, explain these physical phenomena.

The SM itself can provide information on its validity. This originates from the fact that the possible mass range of the Higgs boson is bounded and that those bounds depend on the cutoff of the theory. The upper bound is related to the triviality of the theory, i.e. the observation that the quartic coupling runs to zero when the cut-off is sent to infinity [3]. The lower Higgs boson mass bound is determined by the requirement, that the electroweak vacuum is stable.

Theoretical developments indicate, that a Higgs boson with a mass below $\approx 129 \mathrm{GeV}$ results in a metastable vacuum [4], although this result is still affected by uncertainties coming mainly from present errors of the strong coupling constant and, in particular, the top quark mass. The scale at which this metastability occurs can be estimated from the evolution of all standard model couplings from the electroweak scale up to the Planck scale. The metastability then results from the fact, that the quartic coupling turns negative at a certain energy scale. These calculations are performed solely within the framework of the SM and no extensions are considered.

As a consequence of triviality the cutoff cannot be removed and the SM needs to be considered as an effective theory only. This allows the inclusion of higher dimensional operators in the theory which can be interpreted as being induced by some physics beyond the SM. A possible minimal extension of the standard model would be, to add a dimension-6 operator, namely a $\left(\phi^{\dagger} \phi\right)^{3}$-term, to the scalar part of the standard model. This term with a positive coupling $\lambda_{6}$ stabilizes the effective potential even in case of negative quartic self coupling.

In this work we investigate the influence of such a dimension-6 operator in the framework of a Higgs-Yukawa model, which is a reduction of the standard model to only consider the complex scalar doublet and quarks. For our computations we will use a lattice regularization of the HiggsYukawa model employing a chirally invariant lattice formulation. Eventually we want to test, how strongly one can alter the lower Higgs boson mass bound and the vacuum structure of the theory, if a $\left(\phi^{\dagger} \phi\right)^{3}$-term is present. Results from an analysis of the renormalization group approach in a $Z_{2}$-symmetric Higgs-Yukawa model suggest, that the mass bound indeed can be decreased [5].

As a first step we will map out the phase structure of the model in the presence of such a $\lambda_{6}\left(\phi^{\dagger} \phi\right)^{3}$-term, since the more complicated Higgs potential may lead to additional phase transitions which can put bounds on the parameters of the theory. The exploration of the phase diagram will be carried out non-perturbatively by means of numerical simulations and the results will be compared to (lattice) perturbative calculations of the constraint effective potential. 


\section{Higgs-Yukawa model and implementation}

The field content of the Higgs-Yukawa model is given by a fermion doublet $\Psi=(t, b)^{T}$ and the scalar complex doublet $\varphi$. We restrict ourselves to the simple case of one fermion doublet with mass degenerate quarks even though in principle a more general case would be possible but significantly more computertime expensive. In the continuum notation the action is given by:

$$
\begin{aligned}
S^{\operatorname{cont}}[\bar{\psi}, \psi, \varphi]=\int d^{4} x\left\{\frac{1}{2}\left(\partial_{\mu} \varphi\right)^{\dagger}\left(\partial^{\mu} \varphi\right)\right. & \left.+\frac{1}{2} m_{0}^{2} \varphi^{\dagger} \varphi+\lambda\left(\varphi^{\dagger} \varphi\right)^{2}+\lambda_{6}\left(\varphi^{\dagger} \varphi\right)^{3}\right\} \\
& +\int d^{4} x\left\{\bar{t} \not \partial t+\bar{b} \not \partial b+y\left(\bar{\psi}_{L} \varphi b_{R}+\bar{\psi}_{L} \tilde{\varphi} t_{R}\right)+\text { h.c. }\right\},
\end{aligned}
$$

with $\tilde{\varphi}=i \tau_{2} \phi^{*}$ and $\tau_{2}$ being the second Pauli matrix. The bare standard model parameters are given by $m_{0}^{2}$ and $\lambda$ for the Higgs potential and $y$ for the Yukawa coupling. Further, we added the dimension- 6 operator $\lambda_{6}\left(\varphi^{\dagger} \varphi\right)^{3}$ to the action.

For the numerical implementation of this model we us a polynomial hybrid Monte Carlo algorithm [6] with dynamical overlap fermions. Details of the implementation can be found in [7]. On the lattice, it is convenient to rewrite the bosonic part of the action in an Ising model like way:

$$
S_{B}[\phi]=-\kappa \sum_{x, \mu} \phi_{x}^{\dagger}\left[\phi_{x+\mu}+\phi_{x-\mu}\right]+\sum_{x}\left(\phi_{x}^{\dagger} \phi_{x}+\hat{\lambda}\left[\phi_{x}^{\dagger} \phi_{x}-1\right]^{2}+\hat{\lambda}_{6}\left[\phi_{x}^{\dagger} \phi_{x}\right]^{3}\right) .
$$

Here the scalar field is represented as a real four-vector and the relation to the continuum notation is given by:

$$
\varphi(x)=\sqrt{2 \kappa}\left(\begin{array}{c}
\phi_{x}^{2}+i \phi_{x}^{1} \\
\phi_{x}^{0}-i \phi_{x}^{3}
\end{array}\right), \quad m_{0}^{2}=\frac{1-2 \hat{\lambda}-8 \kappa}{\kappa}, \quad \lambda=\frac{\hat{\lambda}}{4 \kappa^{2}}, \quad \lambda_{6}=\frac{\hat{\lambda}_{6}}{8 \kappa^{3}} .
$$

For the determination of the phase structure, we employ the magnetization $m$ as order parameter. The magnetization is given by the modulus of the average scalar field and is related to its vacuum expectation value (vev) via:

$$
m=\left\langle\left|\frac{1}{V} \sum_{x} \phi_{x}\right|\right\rangle, \quad v e v=\sqrt{2 \kappa} \cdot m
$$

\section{Constraint effective potential}

To compare our numerical results to perturbation theory we employ the constraint effective potential (CEP) $[8,9]$. The basic idea is that the potential $U(\hat{v})$ that only depends on the zero mode $\hat{v}$ of the scalar field corresponding to its vacuum expectation value (vev). If one assumes that the groundstate of the system is at a non-vanishing vev, the scalar doublet can be decomposed into the Higgs mode and three goldstone modes. The perturbative calculations are done by explicitly keeping the lattice regularisation, i.e. for the fermionic determinant the overlap operator is used and all sums over lattice momenta are performed numerically. A derivation of the constrained effective potential used here can be found in [10]. The CEP up to the first order in $\lambda$ and $\lambda_{6}$ is given by:

$$
\begin{aligned}
U(\hat{v})= & U_{f}(\hat{v})+\frac{m_{0}^{2}}{2} \hat{v}^{2}+\lambda \hat{v}^{4}+\lambda_{6} \hat{v}^{6} \\
& +\lambda \cdot \hat{v}^{2} \cdot 6\left(P_{H}+P_{G}\right)+\lambda_{6} \cdot\left(\hat{v}^{2} \cdot\left(45 P_{H}^{2}+54 P_{G} P_{H}+45 P_{G}^{2}\right)+\hat{v}^{4} \cdot\left(15 P_{H}+9 P_{G}\right)\right) .
\end{aligned}
$$


The fermionic contribution $U_{f}$ comes from integrating out the fermions in the background of a constant field:

$$
U_{f}(\hat{v})=-\frac{4}{V} \sum_{p} \log \left|v(p)+y \cdot \hat{v} \cdot\left(1-\frac{1}{2 \rho}\right) v(p)\right|^{2},
$$

with $v(p)$ denoting the eigenvalues of the overlap operator corresponding to the momentum $p$ :

$$
v(p)=\rho\left(1+\frac{i \sqrt{\tilde{p}^{2}}+r \hat{p}^{2}-\rho}{\sqrt{\tilde{p}^{2}+\left(r \hat{p}^{2}-\rho\right)^{2}}}\right), \quad \hat{p}^{2}=4 \sum_{\mu} \sin ^{2}\left(\frac{p_{\mu}}{2}\right), \quad \tilde{p}^{2}=\sum_{\mu} \sin ^{2}\left(p_{\mu}\right) .
$$

The propagator sums for the Higgs and the Goldstone bosons are given by:

$$
P_{H}=\frac{1}{V} \sum_{p \neq 0} \frac{1}{\hat{p}^{2}+m_{H}^{2}}, \quad P_{G}=\frac{1}{V} \sum_{p \neq 0} \frac{1}{\hat{p}^{2}} .
$$

We note, that even though we use the continuum notation, all quantities are meant to be dimensionless, i.e. the lattice spacing is set to one implicitly. The vev is given by the minimum of the potential, and by setting it to the phenomenologically known value of vev of $246 \mathrm{GeV}$ one obtains a physical scale in this approach:

$$
\left.\frac{\mathrm{d} U(\hat{v})}{\mathrm{d} \hat{v}}\right|_{\hat{v}=v e v} \stackrel{!}{=} 0, \quad \Lambda=\frac{246 \mathrm{GeV}}{v e v} .
$$

Further, the squared Higgs boson mass $m_{H}^{2}$ is determined by the curvature of the potential at its minimum:

$$
\left.\frac{\mathrm{d}^{2} U(\hat{v})}{\mathrm{d} \hat{v}^{2}}\right|_{\hat{v}=v e v}=m_{H}^{2}
$$

Due to the explicit appearence of the Higgs boson mass in the propagator sum (3.4), we have to use an iterative approach to solve the CEP. To this end, we fix the parameters $m_{0}^{2}, y, \lambda$ and $\lambda_{6}$ and iterate eqs. $(3.5,3.6)$ until we find convergence.

\section{Results}

As already mentioned, this work is a first step towards a systematic investigation whether it is possible to alter the lower Higgs boson mass bound if in addition to the ordinary standard model Higgs potential a dimension-6 operator $\lambda_{6}\left(\phi^{\dagger} \phi\right)^{3}$ is included. To this end, we have to map out the bulk (non-thermal) phase structure. The question is where phase transitions occur and of which order these phase transitons are. In order to separate the cut-off scale from the low-energy quantities, we have to search for 2 nd order phase transitions. On the other hand, it is natural to expect the appearance of 1st order phase transitions in the presence of the dimension- 6 operator. We would like to be certain that our study of the Higgs-boson mass bounds is performed away from these 1st order phase transitions. Locating the tri-critical line in the $\lambda-\lambda_{6}$ plane is then the first task of our investigation. In all calculations we will keep the Yukawa couplings fixed to obtain the physical top quark mass for which we follow ref. [11] and use the tree-level relation

$$
y=\frac{m_{t}}{v e v}=\frac{175 \mathrm{GeV}}{246 \mathrm{GeV}} .
$$


For our study we have employed two values of the coupling $\lambda_{6}$ and for each we choose a set of values for $\lambda \leq 0$. With the Yukawa coupling fixed according to (4.1) ${ }^{1}$, there is only one free parameter left, namely $m_{0}^{2}$ which is directly related to $\kappa$ according to (2.3). We then perform scans in $\kappa$ to determine regions of broken and symmetric phases corresponding to clearly non-zero and almost zero values of the magnetization.

In figure 1 we show results comparing curves obtained from numerical evaluations of the CEP (3.1) and direct numerical simulations performed on rather small lattices of volume $V=12^{3} \times 24$. For both tested values of $\lambda_{6}$ depending on the choice of $\lambda$ there is quite clear evidence for either second or first order phase transitions.

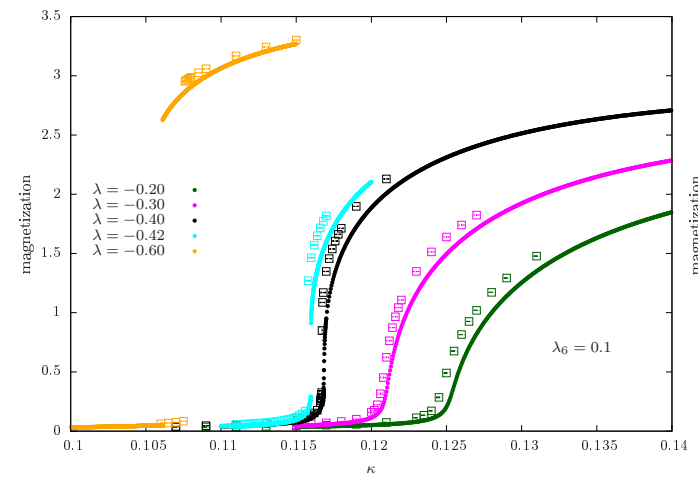

(a) $\lambda_{6}=0.10$

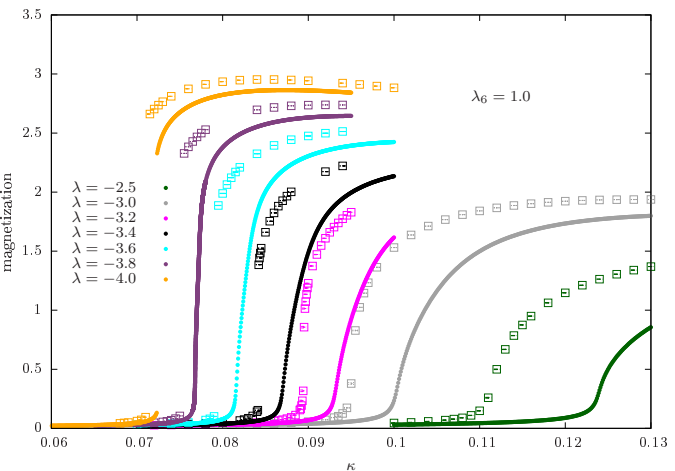

(b) $\lambda_{6}=1.00$

Figure 1: These plots show the magnetization as it is obtained in simulations (open squares) and from the CEP (dots) for two values of $\lambda_{6}$. Both, the simulations and the evaluation of the CEP were done on $12^{3} \times 24$ lattices. The left plot shows results for a rather small value of $\lambda_{6}=0.1$ and the agreements between perturbative and non-perturbative determination is quite good, whereas the agreement for the non-perturbative case of $\lambda_{6}=1$ can only be considered as qualitative.

To test, whether some of the transitions we observe are of first order, we show the Monte Carlo time trajectories for the magnetization for a chosen set of $\kappa$-values in figure 2 . There three neighboring kappa values are shown and one can observe, that while the smallest and the largest values of $\kappa$ lead to stable runs, the middle value shows metastabilities where the system jumps between two values of the magnetization. It is also possible, to access the CEP from the simulation data. Up to a constant, the CEP can be related to the logarithm of the histogram of the magnetization, see e.g. [12].

To address the question how the addition of the $\lambda_{6}$-term alters the Higgs mass bounds, we are so far restricted to the mass determination from the CEP. The standard model lower Higgs boson mass bound is obtained by setting $\lambda$ to zero [11]. If a $\lambda_{6}$ coupling is switched on, we find a behaviour of the Higgs boson mass as shown in figure 3 for $\lambda_{6}=0.05$ and 0.1 . In both cases, it is possible, to find (negative) values for $\lambda$, that allow a significant decrease of the Higgs boson mass for intermediate cutoffs without entering the region of first order phase transitions discussed above. In fig. 3 we show finite volume, but also the infinite volume curves demonstrating that that the shift in the mass survives the infinite volume limit.

\footnotetext{
${ }^{1}$ The couplings $\lambda$ and $\lambda_{6}$ in this section are the dimension-less couplings in the continuum notation and may not to be confused with the parameters being rescaled by powers of $\sqrt{2 \kappa}$
} 


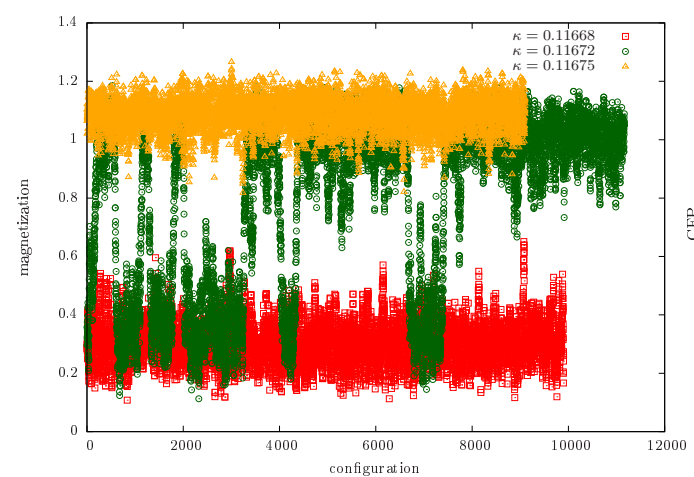

(a) trajectories

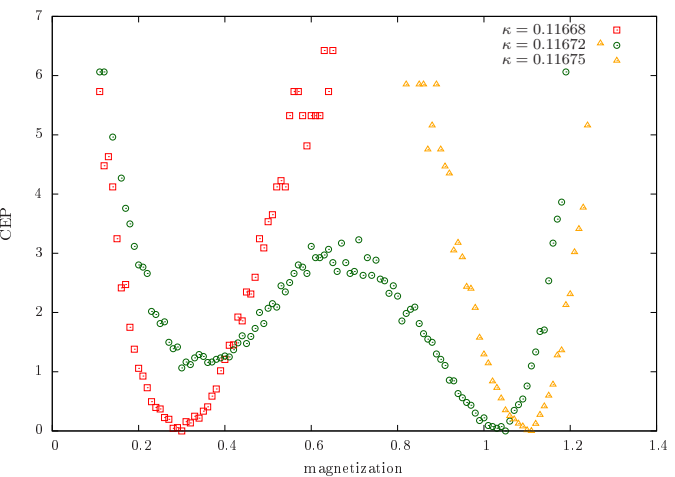

(b) CEP from simulation

Figure 2: The left plot shows the Monte Carlo time trajectories of the magnetization corresponding to simulation data for $\lambda=-0.4$ from fig. 1a where one observes a typical metastable behaviour for $\kappa=0.11672$ with the magnetization jumping between two values. The runs of adjacent $\kappa$ do not show this behaviour. The right plot shows the CEP as it is obtained from the simulation for those three values of $\kappa$. Both plots nicely indicate the existence of a first order phase transition.
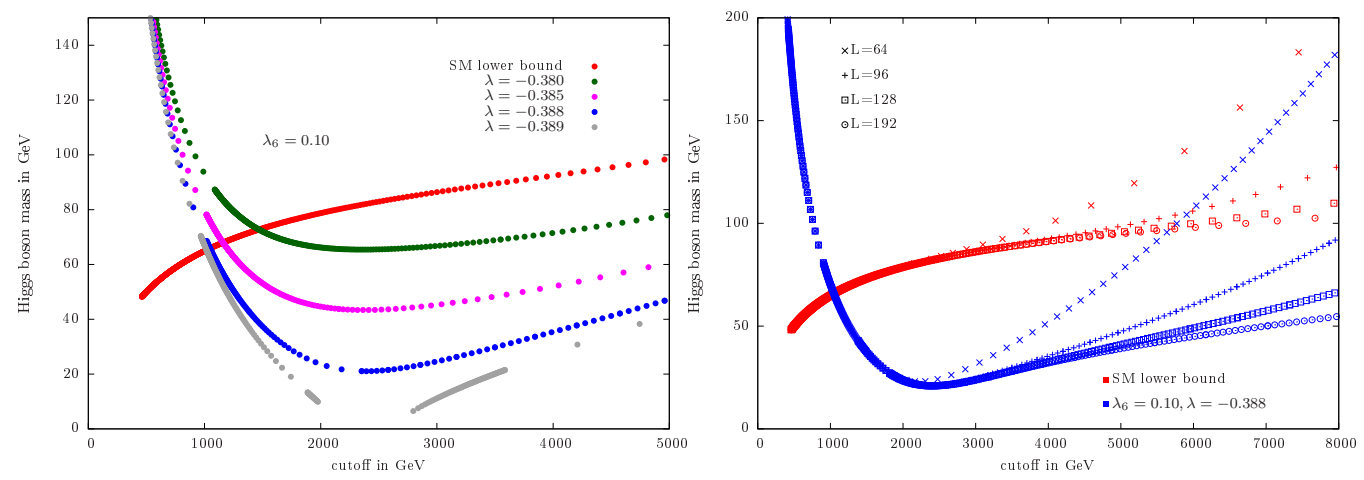

Figure 3: Here we show the dependence of the Higgs boson mass on the cutoff as it is obtained in the CEP. The left plot compares the results for various values of $\lambda$ on a $96^{3} \times 192$ lattice while keeping $\lambda_{6}=0.1$ constant. Additionally we show the standard model lower bound indicated by the red points. The gap in the gray data points originates from the first order phase transition. The right plot shows the volume dependence for various $L^{3} \times 2 L$ lattices while keeping $\lambda=-0.388$ and $\lambda_{6}=0.1$ fixed. It also shows the volume dependence of the standard model mass bound.

\section{Conclusions and outlook}

In this work, we have added a dimension-6 operator to a Higgs-Yukawa model to test the stability of a so extended SM. We found that for fixed values of $\lambda_{6}=0.1$ and for a cutoff of about $\gtrsim 1.5 \mathrm{TeV}$, the Higgs boson mass can be lowered when the quartic coupling is driven more and more negative, as was also found in ref. [5]. In addition, we detected that for a certain (negative) value of the quartic coupling the transition between the symmetric and the broken phase turns first order and the separation between the cut-off and the low-energy scale is lacking, leading to an absolute lower bound of the Higgs boson mass. With this we conclude that for the here considered value of a $\lambda_{6}$ coupling a Higgs boson mass of $126 \mathrm{GeV}$ is fully compatibale with an addition of a 
$\phi^{6}$ term. In addition, since such a term is quadratically suppressed with the cutoff, it has no effect at very high energies.

An interesting question remains, however, namely what happens at very large values of $\lambda_{6}=$ $O(10)$. Will there be clash with the Higgs boson mass value leading to bounds of the $\lambda_{6}$ coupling like in the case of our fourth fermion investigation [13]. Or, can it even be possible that are a low values of the cutoff of $\mathrm{O}(10 \mathrm{TeV})$ a metastable behaviour can be found? We plan to address these question in the future by studying larger values of the $\lambda_{6}$ coupling.

\section{Acknowledgement}

Simulations have been performed at the SGI system HLRN-II at the HLRN supercomputing service Berlin-Hannover and the PAX cluster at DESY-Zeuthen. This work is supported by Taiwanese NSC via the grant 102-2113-M-009-002-MY3 and by the DFG through the DFG-project Mu932/4-4.

\section{References}

[1] Georges Aad et al. Measurements of Higgs boson production and couplings in diboson final states with the ATLAS detector at the LHC. Phys.Lett., B, 2013.

[2] Serguei Chatrchyan et al. Observation of a new boson with mass near $125 \mathrm{GeV}$ in pp collisions at $\sqrt{s}$ $=7$ and 8 TeV. JHEP, 1306:081, 2013.

[3] Roger F. Dashen and Herbert Neuberger. How to Get an Upper Bound on the Higgs Mass. Phys.Rev.Lett., 50:1897, 1983.

[4] Giuseppe Degrassi, Stefano Di Vita, Joan Elias-Miro, Jose R. Espinosa, Gian F. Giudice, et al. Higgs mass and vacuum stability in the Standard Model at NNLO. JHEP, 1208:098, 2012.

[5] Holger Gies, Clemens Gneiting, and René Sondenheimer. Higgs Mass Bounds from Renormalization Flow for a simple Yukawa model. 2013.

[6] Roberto Frezzotti and Karl Jansen. The PHMC algorithm for simulations of dynamical fermions: 1. Description and properties. Nucl.Phys., B555:395-431, 1999.

[7] P. Gerhold. Upper and lower Higgs boson mass bounds from a chirally invariant lattice Higgs-Yukawa model.

[8] R. Fukuda and E. Kyriakopoulos. Derivation of the Effective Potential. Nucl.Phys., B85:354, 1975.

[9] L. O'Raifeartaigh, A. Wipf, and H. Yoneyama. The Constraint Effective Potential. Nucl.Phys., B271:653, 1986.

[10] P. Gerhold and K. Jansen. The Phase structure of a chirally invariant lattice Higgs-Yukawa model numerical simulations. JHEP, 0710:001, 2007.

[11] P. Gerhold and K. Jansen. Lower Higgs boson mass bounds from a chirally invariant lattice Higgs-Yukawa model with overlap fermions. JHEP, 0907:025, 2009.

[12] Igor Dimitrovic, Jurg Nager, Karl Jansen, and Thomas Neuhaus. Shape of the constraint effective potential: A Monte Carlo study. Phys.Lett., B268:408-414, 1991.

[13] J. Bulava, K. Jansen, and A. Nagy. Constraining a fourth generation of quarks: non-perturbative Higgs boson mass bounds. Phys.Lett., B723:95-99, 2013. 